\title{
Temporal Dynamics of GABA and Glx in the Visual Cortex
}

\author{
(D)Reuben Rideaux
}

https://doi.org/10.1523/ENEURO.0082-20.2020

Department of Psychology, University of Cambridge, Cambridge CB2 3EB, United Kingdom

\begin{abstract}
Magnetic resonance spectroscopy (MRS) can be used in vivo to quantify neurometabolite concentration and provide evidence for the involvement of different neurotransmitter systems (e.g., inhibitory and excitatory) in sensory and cognitive processes. The relatively low signal-to-noise ratio of MRS measurements has shaped the types of questions that it has been used to address. In particular, temporal resolution is often sacrificed in MRS studies to achieve a signal sufficient to produce a reliable estimate of neurometabolite concentration. Here we apply novel analyses with large datasets from human participants (both sexes) to reveal the dynamics of $\mathrm{GABA}^{+}$and Glx in visual cortex while participants are at rest (with eyes closed) and compare this with changes in posterior cingulate cortex from a previously collected dataset (under different conditions). We find that the dynamic concentration of $\mathrm{GABA}^{+}$and Glx in visual cortex drifts in opposite directions; that is, GABA ${ }^{+}$ decreases while Glx increases over time. Further, we find that in visual, but not posterior cingulate cortex, the concentration of $\mathrm{GABA}^{+}$predicts that of Glx $120 \mathrm{~s}$ later, such that a change in $\mathrm{GABA}^{+}$is correlated with a subsequent opposite change in Glx. Together, these results expose novel temporal trends and interdependencies of primary neurotransmitters in visual cortex. More broadly, we demonstrate the feasibility of using MRS to investigate in vivo dynamic changes of neurometabolites.
\end{abstract}

Key words: GABA glutamate; Glx; magnetic resonance spectroscopy; temporal dynamics; visual cortex

\section{Significance Statement}

Using large datasets of magnetic resonance spectroscopy acquisitions from human participants, we develop novel analyses to investigate the temporal dynamics of neurometabolite concentration in visual cortex. We find that, while participants are at rest, the concentration of $\mathrm{GABA}^{+}$and Glx drifts in opposite directions; that is, $\mathrm{GABA}^{+}$decreases while Glx increases over time. Further, we find that the concentration of $\mathrm{GABA}^{+}$predicts that of Glx 120 s later, such that a change in $\mathrm{GABA}^{+}$is correlated with a subsequent opposite change in Glx. We show that these phenomena are regionally localized to visual cortex.

Received March 4, 2020; accepted June 1, 2020; First published June 22, 2020.

The authors declare no competing financial interests.

Author contributions: R.R. designed research; R.R. performed research;

R.R. analyzed data; R.R. wrote the paper.

This research was supported by Leverhulme Trust Grant ECF-2017-573 and Isaac Newton Trust Grant 17.08(0).

Correspondence should be addressed to Reuben Rideaux at reuben. rideaux@gmail.com.

https://doi.org/10.1523/ENEURO.0082-20.2020

Copyright (C) 2020 Rideaux

This is an open-access article distributed under the terms of the Creative Commons Attribution 4.0 International license, which permits unrestricted use, distribution and reproduction in any medium provided that the original work is properly attributed.

\section{Introduction}

Magnetic resonance spectroscopy (MRS) can be used in vivo to measure the concentration of neurometabolites within the brain. The blood oxygenation leveldependent (BOLD) signal measured using functional magnetic resonance imaging (fMRI) can provide evidence of neural activity; however, MRS can provide evidence that can be used to distinguish between different types of activity (e.g., excitatory and inhibitory). For the purpose of understanding neural mechanisms, identifying the involvement of neurotransmitter systems that support sensory/cognitive processes can be 
more informative than locating regions of neural representation.

Previous work using MRS to understand the role of different neurotransmitters in normal brain function has focused on neurometabolite activity in visual cortex; in particular, the function of $\gamma$-aminobutyric acid (GABA) and glutamate (Glu), the primary inhibitory and excitatory neurotransmitters in the central nervous system, respectively. A consistent finding from these studies is that Glu increases in visual cortex in response to visual stimulation, which has been interpreted as increased involvement of this neurotransmitter during the processing of visual stimuli (Mangia et al., 2007; Lin et al., 2012; Schaller et al., 2013; Bednarõík et al., 2015, 2018; Ip et al., 2017; Kurcyus et al., 2018). The findings relating to GABA have been less consistent. One study found less GABA in visual cortex during visual stimulation compared with baseline (Mekle et al., 2017), while other studies have not replicated this result (Mangia et al., 2007; Schaller et al., 2013; Bednarõík et al., 2015, 2018; Kurcyus et al., 2018).

The classic "static" fMRS approach (i.e., comparing the average neurometabolite concentration during one experimental condition with another) has implicated GABA and Glu in visual processing. However, the information provided using this approach is severely limited. In particular, by reducing the measure of neurometabolite concentration to a single estimate averaged across a viewing condition, dynamic changes in concentration that occur under different states of visual processing are obscured. By contrast, establishing the temporal dynamics in neurometabolite concentration will reveal the timing and magnitude of change in different neurotransmitters and novel relationships between neurotransmitters. This information is essential for a comprehensive understanding of the role of neurotransmitters in visual processing, but, more broadly, it may inform our understanding of the balance between excitation and inhibition, which is thought to be a key factor in multiple neurologic and psychiatric illnesses (Bradford, 1995; Rubenstein and Merzenich, 2003; Kehrer et al., 2008).

The temporal resolution of MRS is highly restrained by the signal-to-noise ratio of measurements acquired using the technique. Although the duration that restricts the temporal resolution of MRS (i.e., the relaxation time) can be similar to that of $\mathrm{AMRI}$ (i.e., $\sim 2 \mathrm{~s}$ ), to yield a reliable measurement of neurometabolite concentration from within the brain, multiple transients must be combined to reach a sufficient signal-to-noise ratio (Mikkelsen et al., 2018). For example, it is common to combine between 200 and 300 transients $(\sim 10 \mathrm{~min})$ to produce a single measure of neurometabolite concentration (Kurcyus et al., 2018; Rideaux and Welchman, 2018). Here we overcome the signal-to-noise limitation of MRS by applying temporal analyses of neurometabolite concentration to a large dataset of participants. We measure the dynamic concentration of GABA and GIx in visual cortex of participants while at rest (with closed eyes). We assess the regional specificity of temporal dynamics by comparing these results with data from posterior cingulate cortex (Mikkelsen et al., 2017, 2019). To observe the temporal dynamics of neurometabolites, we analyze the data in two ways. We first take a moving average of an $\sim 6 \mathrm{~min}$ period, to reveal low-frequency trends in the data (Chen et al., 2017; Rideaux et al., 2019). Next, using a new technique, we combine data across participants (rather than time), which allows us to track the concentration of neurometabolites with relatively high temporal resolution (12 s) over a 13 min period.

Based on previous empirical evidence, we may expect GIx to decrease and GABA to increase under conditions of no visual stimulation. By contrast, our analyses reveal the opposite pattern of results: Glx increases while GABA decreases (in visual, but not posterior cingulate cortex). These results are broadly consistent with findings from visual deprivation studies (Boroojerdi et al., 2000; Lunghi et al., 2015) and may provide a link between the conflicting results from MRS studies that use relatively short periods of visual deprivation compared with those with longer periods. Further, we expose large changes in GABA and Glx, previously obscured by averaging over long durations, and reveal a striking relationship between GABA and GIx in visual cortex: a change in GABA predicts the opposite change in Glx 120 s later.

\section{Materials and Methods}

\section{Participants}

Fifty-eight healthy participants with normal or corrected-to-normal vision participated in the experiment. The mean age was 24.4 years (age range, 19.4-40.5 years; 31 women). Participants were screened for contraindications to MRI before the experiment. All experiments were conducted in accordance with the ethical guidelines of the Declaration of Helsinki and were approved by the university ethics committee, and all participants provided informed consent.

\section{Data collection}

Participants underwent an MR spectroscopic acquisition targeting visual cortex. During the acquisition, the lights in the room were turned off and participants were instructed to close their eyes. To compare these data with those measured from another brain region, we reanalyzed previously gathered MR spectroscopic data targeting posterior cingulate cortex (Mikkelsen et al., 2017, 2019).

\section{Data acquisition}

Magnetic resonance scanning targeting visual cortex was conducted on a $3 \mathrm{~T}$ Siemens Prisma equipped with a 32-channel head coil. Anatomical T1-weighted images were acquired for spectroscopic voxel placement with an MP-RAGE sequence. For the detection of GABA ${ }^{+}$and GIx, spectra were acquired using a MEGA-PRESS sequence (Mescher et al., 1996, 1998): TE $=68 \mathrm{~ms}$; TR $=3000 \mathrm{~ms}$; 256 transients of 2048 data points were acquired in $13 \mathrm{~min}$ experiment time; a $14.28 \mathrm{~ms}$ Gaussian editing pulse was applied at $1.9 \mathrm{ppm}(\mathrm{ON})$ and $7.5 \mathrm{ppm}$ (OFF). Water suppression was achieved using variable power with optimized relaxation delays (Tkáč and Gruetter, 2005) and outer volume suppression. Automated shimming followed 
a

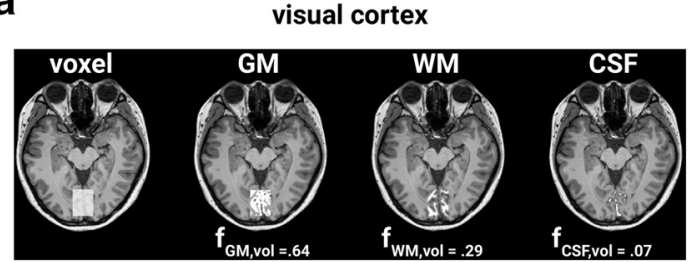

C

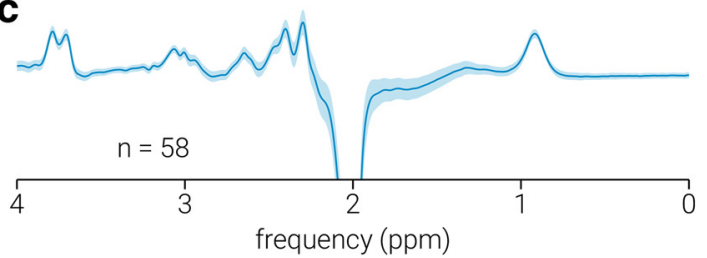

b

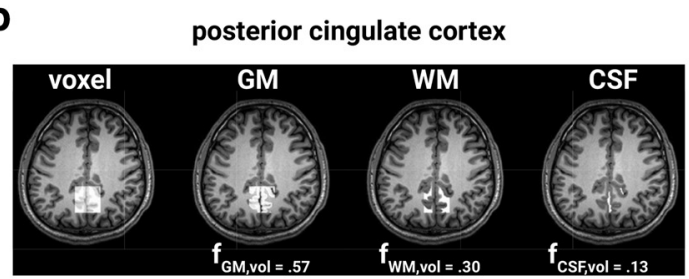

d

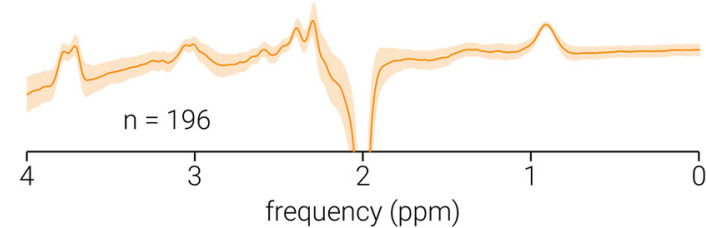

Figure 1. Data acquisition. $\boldsymbol{a}, \boldsymbol{b}, \mathrm{MRS}$ voxel placement for visual (a) and posterior cingulate (b) cortices on a T1-weighted structural image and probabilistic partial volume voxel maps following tissue segmentation for a representative participant. Corresponding tissue proportions of gray matter (GM), white matter (WM), and CSF are shown. $\boldsymbol{c}, \boldsymbol{d}$, Average spectra across all subjects for visual (c) and posterior cingulate $(\boldsymbol{d})$ cortices; the number of subjects comprising each average spectrum is shown, and gray shaded regions indicate SD. Note the nonuniform baseline in $\boldsymbol{d}$, which resulted from the incomplete removal of the water peak (which was suppressed in the visual but not in the posterior cingulate cortex data) in the difference spectra; importantly, this nonuniformity was modeled and removed during neurometabolite quantification.

by manual shimming was conducted to achieve $\sim 12 \mathrm{~Hz}$ water linewidth.

The "Big GABA" dataset comprises a collection of MRS datasets collected by different groups using the same parameters on GE, Phillips, and Siemens scanners. This dataset was acquired separately for a previous study and targeted posterior cingulate cortex; thus, here it was reused as a region specificity control for the main visual cortex data. A detailed description of the data acquisitions for the Big GABA dataset can be found in studies by Mikkelsen et al. $(2017,2019)$. To summarize the procedure, magnetic resonance scanning targeting posterior cingulate cortex was conducted on 3 T Siemens, GE, and Phillips scanners equipped with 8-, 32-, or 64-channel head coils. Spectra were acquired using a MEGA-PRESS sequence: $\mathrm{TE}=68 \mathrm{~ms}$; TR $=2000 \mathrm{~ms} ; 320$ transients of either 2048 or 4096 data points were acquired in a $12 \mathrm{~min}$ experiment time; a $15 \mathrm{~ms}$ Gaussian editing pulse was applied at $1.9 \mathrm{ppm}(\mathrm{ON})$ and $7.5 \mathrm{ppm}$ (OFF). Note that, unlike the data collected in visual cortex, data collected in posterior cingulate cortex was not water suppressed, which can negatively impact the accurate quantification of other metabolites (e.g., sideband artifacts; for a review of issues relating to water suppression, see Dong, 2015). The Big GABA dataset comprises subdatasets collected by different research groups at different facilities. The following subdatasets from the Big GABA dataset were used in the current study: G4, G5, G7, G8, P1, P3, P4, P5, P6, P7, P8, $\mathrm{P} 9, \mathrm{P} 10, \mathrm{~S} 1, \mathrm{~S} 6$, and S8 (the letters in the subdatasets refer to the scanner make and the numbers refer to the group that collected the data). Each subdataset (e.g., G4) comprises data from between 8 and 12 participants; in total there were data from 196 participants. With the exception of $\mathrm{G} 1$ and $\mathrm{G} 6$, this includes all of the publicly available Big GABA datasets. We used the macromolecule unsuppressed transients from these datasets for closer comparison with visual cortex data, and we excluded data from $\mathrm{G} 1$ and $\mathrm{G} 6$ as these had fewer transients.

Spectra were acquired from a location targeting visual cortices (i.e., V1/V2; Fig. 1a), and posterior cingulate cortex (Fig. 1b); note that the posterior cingulate cortex data were acquired from the (previously described) publicly available Big GABA dataset. The voxel targeting visual cortex $(3 \times 3 \times 2 \mathrm{~cm})$ was placed medially in the occipital lobe; the lower face aligned with the cerebellar tentorium and was positioned so as to avoid including the sagittal sinus and to ensure that it remained within the occipital lobe. The voxel targeting posterior cingulate cortex $(3 \times 3$ $\times 3 \mathrm{~cm}$ ) was positioned in the medial parietal lobe and rotated in the sagittal plane to align with a line connecting the genu and splenium of the corpus callosum. The coordinates of the voxel location were used to draw a mask on the anatomic T1-weighted image to calculate the volume of gray matter, white matter, and cerebrospinal fluid (CSF) within each voxel. Segmentation was performed using the Statistical Parametric Mapping toolbox for MATLAB (SPM12; http://www.fil.ion.ucl.ac.uk/spm/).

\section{Data processing}

Spectral quantification was conducted in MATLAB using GANNET version 3.1 (Edden et al., 2014) and inhouse scripts. Frequency, phase, area, and full-width at half-maximum (FWHM) parameters of the creatine $(\mathrm{Cr})$ peak at $3.0 \mathrm{ppm}$ were estimated by fitting a Lorentzian peak to the data and individual spectra with parameter estimates $>3$ SDs from the mean were omitted from further analysis; the remaining spectra were frequency and phase corrected using these parameters. To ensure spectral alignment between transients across time and participants, spectra were aligned such that the $\mathrm{Cr}$ peak was centered at the same frequency $(3.0 \mathrm{ppm})$. Total creatine (tCr) and total $\mathrm{N}$-acetylaspartate (tNAA) signal intensity 
were determined by fitting a single Lorentzian peak to the mean OFF spectra at 3.0 and $2.0 \mathrm{ppm}$, respectively. ON and OFF spectra were subtracted to produce the edited spectrum (Fig. 1c,d), from which $\mathrm{GABA}^{+}(\mathrm{GABA}$ and coedited macromolecules; 3 ppm) and Glx [a complex comprising Glu and glutamine (Gln); 3.8 ppm] signal intensity were modeled on single- and double-Gaussian peaks, respectively. All neurometabolite signal intensities were calculated as the area of the fitted peak or peaks.

Data in which the FWHM of any of the quantified neurometabolites ( $\mathrm{tCr}$, tNAA, GABA ${ }^{+}$, or Glx) was $>3$ SDs from the mean across each dataset (e.g., visual/posterior cingulate cortex) were omitted from further analysis. This resulted in omission of data from one participant in the visual cortex dataset and three participants in the posterior cingulate cortex dataset.

Intensities of $\mathrm{GABA}^{+}, \mathrm{Glx}$, and tNAA were normalized to the commonly used internal reference $\mathrm{tCr}$ (Jansen et al., 2006), yielding relative concentration values (i.e., GABA ${ }^{+}$: $\mathrm{tCr}$, Glx:tCr, and tNAA:tCr; Fig. 1e). The tCr signal is acquired within the same MEGA-PRESS transients as the target neurometabolites. Thus, normalization of $\mathrm{GABA}^{+}$, Glx, and tNAA to tCr minimizes the influence of changes that occur during the acquisition, which alter the entire spectrum (e.g., changes in signal strength, line width, chemical shift displacement, or dilution associated with changes in blood flow; Ip et al., 2017). For correlational analyses reported in Table 2, a CSF tissue correction (Harris et al., 2015) was applied to the neurometabolite measurements with the following equation:

$$
C_{\text {tisscorr }}=\frac{C_{\text {meas }}}{\left(1-f_{\text {csf }}\right)},
$$

where $C_{\text {tisscorr }}$ and $C_{\text {meas }}$ are the tissue-corrected and uncorrected neurometabolite concentrations (e.g., GABA ${ }^{+}$: $\mathrm{tCr}$ ), respectively, and $f_{\mathrm{csf}}$ is the proportion of CSF within the voxel. All other analyses report concentrations as a proportion of their initial magnitude, and thus do not require tissue correction.

\section{Low-resolution dynamic analysis}

For the low-resolution dynamic analysis of the visual cortex data, we used a sliding window (width, 128 transients; step size, 2 transients) to measure average neurometabolite concentration as it changed over the course of the scan (256 transients/768 s). This window size was based on previous work using this analysis on similar data (Rideaux et al., 2019). For the posterior cingulate cortex data (320 transients/640 s), we matched the duration of the sliding window width to that used for the visual cortex data by including more transients (width, 192 transients; step size, 2 transients).

To determine whether the average change in neurometabolite concentration was significantly different from zero, a two-sided $t$ test $(\alpha, 0.05)$ was performed at each time point. That is, for each brain region, each $t$ test included a single estimate from each participant, which corresponded to the participant's change in neurometabolite concentration at that time. As multiple tests (65) were conducted for each neurometabolite, to reduce the likelihood of spurious significant differences in the time course, a cluster correction was applied at the group level, where time was the clustered dimension. Clusters were defined by the sum of their constituent $t$ values and compared with a null hypothesis distribution of clusters produced by shuffling the time labels (5000 permutations); positive and negative $t$ value clusters were treated separately. Clusters below the 95th percentile of the null hypothesis distribution were disregarded.

\section{High-resolution dynamic analysis}

The temporal resolution of MRS is severely limited by the signal-to-noise ratio of individual spectra. That is, to achieve the signal-to-noise ratio required to yield an accurate neurometabolite measurement, many individual spectra must be combined. To achieve a sufficiently high signal in the low-resolution dynamic analysis, we combined multiple spectra (128) within the same subject. This method produces a dynamic trace for each participant; however, the smoothing produced by the sliding window approach may obscure both the true magnitude of metabolic change over time and dynamic changes occurring at higher frequencies. Thus, to achieve higher temporal resolution, we averaged individual ON and OFF spectra across participants to produce a single trace, with no smoothing, for each condition. As in the low-temporal resolution analysis, we matched the temporal resolution between datasets in the high-temporal resolution analysis (12 s) by using a resolution of four and six transients in the visual and posterior cingulate cortices, respectively.

To test for predictive relationships between $\mathrm{GABA}^{+}$and Glx, we ran a cross-correlation analysis between the above-mentioned high-resolution neurometabolite traces. We tested for relationships in both directions; that is, whether $\mathrm{GABA}^{+}$concentration predicts Glx concentration and vice versa. The neurometabolite traces comprise a limited number of time points (visual cortex, 64; posterior cingulate cortex, 53), and there is an inverse relationship between the lag separating the neurometabolites and the number of time points included in the cross-correlation analysis. This results in less reliable correlation values at lags close to the maximum duration of the neurometabolite traces due to insufficient sample sizes. To avoid these unreliable correlations, we only included lags with a minimum of 25 time points in the analysis (Bonett and Wright, 2000). This yielded a total of 40 correlations for each predictive direction between $\mathrm{GABA}^{+}$and $\mathrm{Glx}$ in visual cortex and 29 for posterior cingulate cortex. Given that multiple tests were conducted, to reduce the likelihood of spurious significant correlation values in the cross-correlation analyses, a cluster correction was applied. Clusters were defined by the sum of their constituent $t$ values and compared with a null hypothesis distribution of clusters produced by shuffling the time labels (5000 permutations); positive and negative $t$ value clusters were treated separately. Clusters below the 95th percentile of the null hypothesis distribution were disregarded.

\section{Significance testing}

The significance of differences between data from different voxel locations was assessed using the 
Table 1: Measures of spectral quality and fit error

\begin{tabular}{|c|c|c|c|c|c|c|c|c|c|}
\hline \multirow[b]{2}{*}{ Location } & \multicolumn{4}{|c|}{ FWHM (Hz) } & \multirow[b]{2}{*}{ Frequency drift (ppm SD) } & \multicolumn{4}{|c|}{ Fit error (\%) } \\
\hline & $\mathrm{GABA}^{+}$ & Glx & tNAA & $\mathrm{tCr}$ & & $\mathrm{GABA}^{+}$ & Glx & tNAA & $\mathrm{tCr}$ \\
\hline$\overline{\mathrm{VC}}$ & $24.9 \pm 1.7$ & $17.8 \pm 0.4$ & $8.4 \pm 0.9$ & $8.5 \pm 0.7$ & $0.0088 \pm 0.0044$ & $7.6 \pm 2.1$ & $1.3 \pm 0.3$ & $3.3 \pm 0.3$ & $3.6 \pm 0.2$ \\
\hline PCC & $20.5 \pm 1.7$ & $16.8 \pm 0.7$ & $8.2 \pm 0.8$ & $7.6 \pm 0.7$ & $0.0065 \pm 0.0061$ & $6.9 \pm 1.7$ & $2.1 \pm 1.5$ & $3.1 \pm 1.3$ & $4.5 \pm 0.5$ \\
\hline
\end{tabular}

Values indicate across-subject averages \pm SD. PCC, Posterior cingulate cortex; VC, visual cortex.

independent-samples $t$ test, and the significance of changes in neurometabolite concentration (from zero change) was assessed using the one-sample $t$ test; all tests were two sided and used an $\alpha=0.05$. The normality assumption was tested with the Shapiro-Wilk test of normality. For data in which the assumption of normality was violated, significance was assessed using the Wilcoxon rank-sum test. The significance of correlations between neurometabolite concentrations was assessed using the Pearson linear correlation and the Pearson linear partial correlation; all tests used an $\alpha=0.05$.

\section{Results}

\section{Spectra quality}

Table 1 shows the average FWHM, frequency drift and fit error for measurements taken from visual and posterior cingulate cortices. For each subject, the FWHM was calculated from the average spectra [i.e., spectra averaged across all transients (256 of 320)]. Frequency drift was calculated as the SD of the position of the Cr peak across individual OFF spectra, before alignment; the frequency drift values shown in Table 1 reflect the average SD across participants. The fit errors for GABA ${ }^{+}$, Glx, tNAA, and $\mathrm{tCr}$ were divided by the amplitude of their fitted peaks to produce normalized measures of uncertainty. The average fit error for each neurometabolite was relatively low (Mullins et al., 2014; Table 1). Comparison between data from visual and posterior cingulate cortices revealed higher FWHM estimates for all quantified neurometabolites in visual cortex $\left(\mathrm{GABA}^{+}: t_{(248)}=16.80, p=8.4 \times 10^{-43}\right.$; Glx: $z=9.24, p=9.4 \times 10^{-20} ;$ tNAA: $z=2.06, p=0.040 ; \mathrm{tCr}:$ $z=7.07, p=1.5 \times 10^{-12}$ ). This suggests that shimming may have been more effective in the posterior cingulate dataset; however, differences in voxel size, voxel tissue composition, and the number of transients collected may have contributed to this difference. We also found that frequency drift $\left(z=5.16, p=2.5 \times 10^{-7}\right)$, and fit errors for $\mathrm{GABA}^{+}(z=2.59, p=0.010)$ and tNAA $(z=4.89$, $\left.p=1.0 \times 10^{-6}\right)$ were higher in the visual cortex dataset.
By comparison, fit error was lower in visual cortex for Glx $\left(z=-5.15, p=2.6 \times 10^{-7}\right)$ and $\operatorname{tCr}(z=-10.92$, $\left.p=9.3 \times 10^{-28}\right)$.

\section{Static analysis}

Figure 2 shows the distribution of gray matter, white matter, and CSF voxel composition across participants for the visual and posterior cingulate cortex datasets. We found that visual cortex voxels were composed of less gray matter $\left(t_{(248)}=7.53, p=9.3 \times 10^{-13}\right)$ and more CSF $\left(z=-6.27, p=3.6 \times 10^{-10}\right)$ than those from posterior cingulate cortex; no significant difference was found between white matter $\left(t_{(248)}=0.27, p=0.787\right)$. We quantified the concentrations of $\mathrm{GABA}^{+}$, GIx, and tNAA using the classic static approach, in which all transients are averaged together to extract a single estimate. After applying a CSF tissue correction, we compared the average neurometabolite concentration across participants between visual and posterior cingulate cortices. We found lower concentrations of $\mathrm{GABA}^{+}: \mathrm{tCr}\left(t_{(248)}=-6.53, p=3.7 \times\right.$ $\left.10^{-10}\right)$ and Glx:tCr $\left(z=-9.33, p=1.0 \times 10^{-20}\right)$ in visual cortex, but a higher concentration of tNAA:tCr $\left(t_{(248)}=\right.$ $4.62, p=6.1 \times 10^{-6}$; Fig. 2).

\section{Low-resolution temporal dynamics of GABA and GIx}

Using a sliding temporal window analysis, we quantified the change in the concentration of $\mathrm{GABA}^{+}$and Glx measured from MRS voxels targeting visual and posterior cingulate cortices over the course of 13 and 12 min periods, respectively. We found that in visual cortex, $\mathrm{GABA}^{+}$significantly decreased (maximum difference $=-5.0 \%, t_{(56)}=$ $-3.25, p=0.002$ ), while Glx significantly increased (maximum difference $=2.7 \%, t_{(56)}=3.74, p=4.4 \times 10^{-4}$ ) over the course of the period (Fig. 3a). By comparison, we found that in posterior cingulate cortex there was no significant change in either GABA or Glx (Fig. 3b). The FWHM of the neurometabolite peaks indicates that spectra from posterior cingulate cortex had better signal quality; further, this dataset contained more than three times

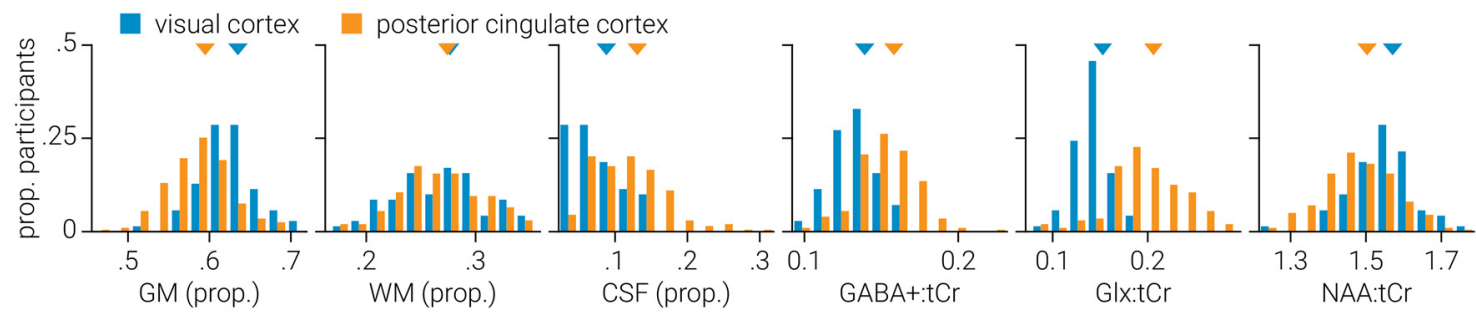

Figure 2. Static analysis. Distribution of gray matter (GM), white matter (WM), and CSF voxel tissue proportions, and neurometabolite $\left(\mathrm{GABA}^{+}\right.$, Glx, tNAA) concentrations across participants for visual and posterior cingulate cortices. Neurometabolite concentrations are tissue corrected and expressed as a ratio to tCr. Triangles indicate distribution means. 
a

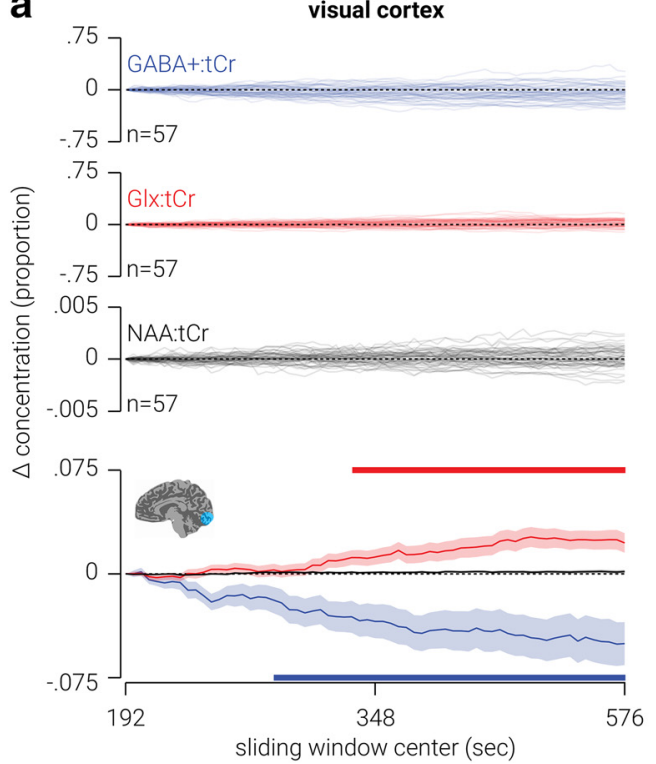

b
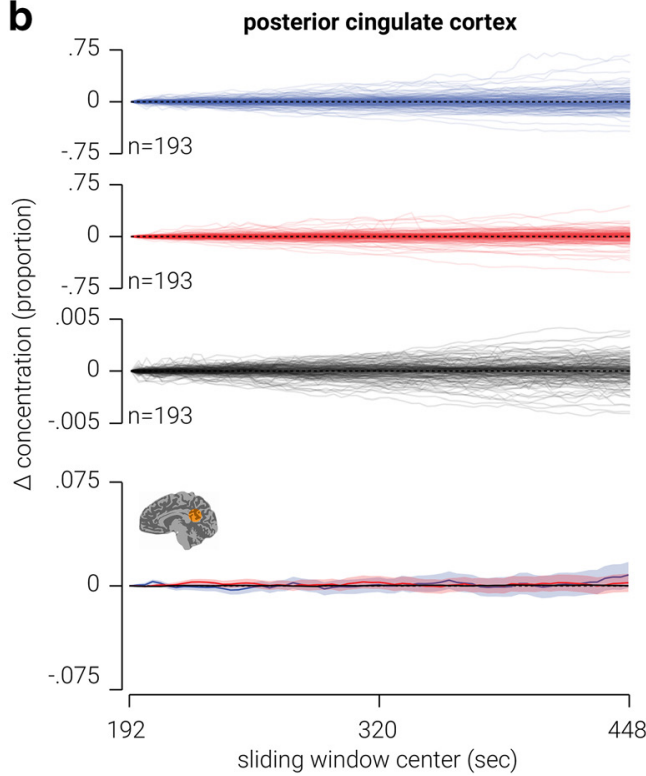

Figure 3. Low-resolution temporal dynamics of neurometabolites in visual and posterior cingulate cortices. a, Top, Individual traces showing change in $\mathrm{GABA}^{+}$, GIx, and tNAA (all referenced to $\mathrm{tCr}$ ) measured from an MRS voxel targeting visual cortex. Bottom, Same as top, but averaged across participants. $\boldsymbol{b}$, Same as $\boldsymbol{a}$, but from a voxel targeting posterior cingulate cortex. Shaded regions indicate SEM, and horizontal colored bars at the top and bottom of $\boldsymbol{a}$ indicate (cluster-corrected) periods of significant difference from zero. Note, the scale of change was smaller for tNAA:tCr than the other two neurometabolites, so it is closely overlaid with the dashed "zero" line.

as many participants as the visual cortex dataset. Thus, it seems unlikely that the neurometabolite drift we observed in visual cortex was also present in posterior cingulate cortex, but that we failed to detect it because of poor signal quality or lack of statistical power. A more parsimonious explanation is that the neurometabolite drift in visual cortex was regionally specific. Given that we referenced $\mathrm{GABA}^{+}$and Glx to tCr, a possible concern is that the changes in neurometabolite concentration observed in visual cortex reflect changes in $\mathrm{tCr}$, as opposed to $\mathrm{GABA}^{+}$or Glx. However, this is unlikely as we did not find any change in tNAA concentration, for either visual or posterior cingulate cortices, which was also referenced for $\mathrm{tCr}$ (Fig. 2a).

We next assessed whether the fit error of the GABA ${ }^{+}$, GIx, tNAA, and tCr peaks changed over the course of the acquisition. For visual cortex, there were no significant changes in the fit error of the tNAA or tCr peaks. However, there were significant increases in fit error for $\mathrm{GABA}^{+}$between 360 and 394 s, and for Glx between 564 and 576 s. A possible concern is that the changes we observed in the concentrations of $\mathrm{GABA}^{+}$and Glx in visual cortex were due to reduced neurometabolite quantification accuracy (i.e., increased fit error). To test this possibility, we assessed the interindividual relationship between change in fit error and change in neurometabolite concentration, for $\mathrm{GABA}^{+}$and Glx in visual cortex. We found no evidence for a relationship between fit error and concentration for either neurometabolite $\left(\mathrm{GABA}^{+}: n=57\right.$, Pearson $r=-0.14, p=0.308$; Glx: $n=57$, Pearson $r=0.06, p=0.676$ ), indicating that change in quantification accuracy does not explain the neurometabolite drift we observed in visual cortex. For posterior cingulate cortex, we found no dynamic change in fit error for any of the neurometabolites, with the exception of the $\mathrm{GABA}^{+}$ peak, where there was a window between 196 and $208 \mathrm{~s}$ in which the fit error was significantly reduced.

Another possible concern is that changes observed in the difference spectra over time are related to scanner field drift due to gradient cooling (Lange et al., 2011) or participant motion (Bhattacharyya et al., 2007). In particular, if the scanner field drifts, the position of the editing pulse relative to the GABA and Glx peaks changes. This may change the efficiency with which the peaks are edited and thus their magnitude in the difference spectrum. As the frequency drift of the visual cortex spectra was higher than that in posterior cingulate cortex, where we did not observe any change in neurometabolite concentration, this may account for the changes in neurometabolite concentration observed in visual cortex. As a test of this possibility, we measured the negative tNAA signal in the difference spectra using an inverse Lorentzian. Like the magnitude of the $\mathrm{GABA}^{+}$and Glx peaks, the magnitude of the negative tNAA signal reflects the efficiency of the editing pulse. Thus, if scanner drift is responsible for changes in the magnitude of the $\mathrm{GABA}^{+}$or Glx peaks, we would expect to see corresponding changes in the amplitude of the edited negative tNAA signal. However, we found no evidence for change in the amplitude of the edited negative INAA signal over time. As a further test of this possibility, we assessed whether there was an interindividual relationship between the degree of frequency drift and change in either $\mathrm{GABA}^{+}$or Glx. If scanner drift produced the change in neurometabolite concentration we observed in visual cortex, we would expect these measures to be positively correlated. By contrast, we found no relationship between frequency drift and either change in $\mathrm{GABA}^{+}(n=57$, Pearson $r=-0.10, p=0.457)$ or 
Table 2: Correlation coefficients between neurometabolites measured from visual and posterior cingulate cortices

\begin{tabular}{|c|c|c|c|c|c|c|}
\hline Location & $\begin{array}{l}\mathrm{GABA}^{+} \\
\text {and } \mathrm{Glx}\end{array}$ & $\begin{array}{l}\text { GABA }^{+} \\
\text {and tNAA }\end{array}$ & $\begin{array}{l}\text { Glx } \\
\text { and tNAA }\end{array}$ & $\begin{array}{l}\Delta \mathrm{GABA}^{+} \\
\text {and } \Delta \mathrm{Glx}\end{array}$ & $\begin{array}{l}\Delta \mathrm{GABA}^{+} \\
\text {and } \Delta \mathrm{tNAA}\end{array}$ & $\begin{array}{l}\Delta \mathrm{Glx} \\
\text { and } \Delta \mathrm{tNAA}\end{array}$ \\
\hline$\overline{\mathrm{VC}}$ & -0.16 & $0.33 *$ & $0.33 *$ & 0.06 & 0.10 & -0.17 \\
\hline PCC & $0.25 * * *$ & $0.50 * * *$ & $0.17 *$ & 0.03 & $-0.18 *$ & 0.04 \\
\hline
\end{tabular}

Correlation coefficients are Pearson linear partial coefficients after controlling for the common reference neurometabolite tCr. All neurometabolites are referenced to $\mathrm{tCr}$ and are tissue corrected. PCC, Posterior cingulate cortex; VC, visual cortex. $* p<0.05 ; * * * p<0.001$

Glx ( $n=57$, Pearson $r=0.06, p=0.662)$. These results indicate that scanner drift did not contribute to the changes in neurometabolite concentration.

\section{Correlational analyses of static and low-temporal resolution measurements}

For each voxel location, we assessed whether there were interindividual relationships between different static neurometabolite concentrations or changes in concentration. For static measurements, we averaged across all 256 of 320 transients. The results of the analysis are shown in Table 2. For static neurometabolite concentrations, we found a positive relationship between Glx and tNAA measured from visual and posterior cingulate cortices. We also found a positive correlation between GABA ${ }^{+}$ and tNAA in visual cortex, and between $\mathrm{GABA}^{+}$and $\mathrm{Glx}$ in posterior cingulate cortex. By contrast, the only relationship between changes in the metabolite concentration found was for $\mathrm{GABA}^{+}$and tNAA in posterior cingulate cortex.

\section{High-resolution temporal dynamics of GABA and GIx}

To assess high-temporal resolution dynamics of neurometabolites, we next combined transients across subjects, rather than across time. In visual cortex, we found that the change in $\mathrm{GABA}^{+}$, relative from the first measurement, was primarily negative and ranged from $\pm 15 \%$ from the average concentration, while the change in Glx was primarily positive and ranged from $\pm 10 \%$ (Fig. 4a). These results are consistent with those from the previous analysis, except the higher temporal resolution obtained with the current approach revealed considerably larger changes in neurometabolite concentration than the previous estimates, which were likely obscured by smoothing measurements across the temporal window. In posterior cingulate cortex, we found that the changes in both $\mathrm{GABA}^{+}$and Glx were primarily negative and similar in amplitude (Fig. 4b).

While GABA and Glu are thought to support opposing mechanisms in the CNS (i.e., inhibition and excitation), it seems reasonable to expect interactions between GABA and Glx, which comprises both Glu and Gln. For example, Gln is a primary source of GABA synthesis (Paulsen et al., 1988; Patel et al., 2001; Rae et al., 2003). Indeed, we found that the static concentration of $\mathrm{GABA}^{+}$and GIx were positively related in posterior cingulate cortex. However, we found no evidence for a relationship between the overall changes in these neurometabolites in either the visual or posterior cingulate cortex (Table 2). One reason for this may be that the relationship between these neurometabolites may only be observed at a high temporal resolution, but not averaged across a 6 min period. To test this hypothesis, we used the high-resolution neurometabolite measurements to perform cross-correlation analyses on $\mathrm{GABA}^{+}$and $\mathrm{Glx}$ concentrations.

For visual cortex, we found that the concentration of $\mathrm{GABA}^{+}$predicted that of Glx between 108 and 132 s later [n(time points) $=(55,54,53)$, Pearson $r=(-0.36,-0.31$, $-0.39), p=(0.008,0.024,0.004)$; Fig. $4 c]$. This relationship was negative, that is, a positive/negative change in $\mathrm{GABA}^{+}$predicted a later change in Glx in the opposite direction. By contrast, we found no periods of latency in which Glx predicted the concentration of $\mathrm{GABA}^{+}$. For posterior cingulate cortex, we found no periods of latency in which there was a significant relationship between GABA $^{+}$and GIx (Fig. 4d).

A possible concern is that the relationship between $\mathrm{GABA}^{+}$and Glx was influenced by their common reference neurometabolite (tCr). In particular, as both $\mathrm{GABA}^{+}$ and $\mathrm{Glx}$ were referenced to $\mathrm{tCr}$, the relationship between them could be explained by an autocorrelation in the concentration of tCr. However, we found the same pattern of results when tNAA, rather than $\mathrm{tCr}$, was used as a reference neurometabolite (Fig. 5a). Another possible concern with this new analysis is that the signal-to-noise ratio is insufficient to yield valid measurements (e.g., due to reduced neurometabolite quantification accuracy). To assess the validity of the measurements, we compared the average FWHM and fit error of peaks produced in the high-resolution analysis with those produced in the lowresolution analysis. For visual cortex, we found no evidence for a difference in FWHM for any of the target neurometabolites $\left(\mathrm{GABA}^{+}: z=-0.06, p=0.948\right.$; Glx: $t_{(119)}=$ $-5.08, p=1.4 \times 10^{-6} ;$ tCr: $\left.z=-1.10, p=0.270\right)$. By comparison, fit error was lower for all target neurometabolites $\left(\mathrm{GABA}^{+}: z=2.38, p=0.017\right.$; Glx: $z=4.21, p=2.5 \times 10^{-5}$; tCr: $\left.t_{(119)}=2.24, p=0.027\right)$ modeled in the high-resolution analysis. Similarly, for posterior cingulate cortex, we found no evidence for a difference in either FWHM $\left(\mathrm{GABA}^{+}: z=1.62, p=0.105\right.$; Glx: $t_{(119)}=1.76, p=0.080$; tCr: $z=1.71, p=0.087)$; however, while we found no evidence for a difference in fit error for GABA ( $z=1.32$, $p=0.187)$ and $\operatorname{Glx}(z=-0.55, p=0.581)$, fit error was higher for $\operatorname{tCr}(z=-2.34, p=0.019)$ in the high-resolution analysis. With the exception of the fit error of the $\mathrm{tCr}$ peak in posterior cingulate cortex, these results support the validity of the signal strength and quantification accuracy in the high-resolution analysis.

To further assess the validity of the measurements, we attempted to reproduce the results from the low- 

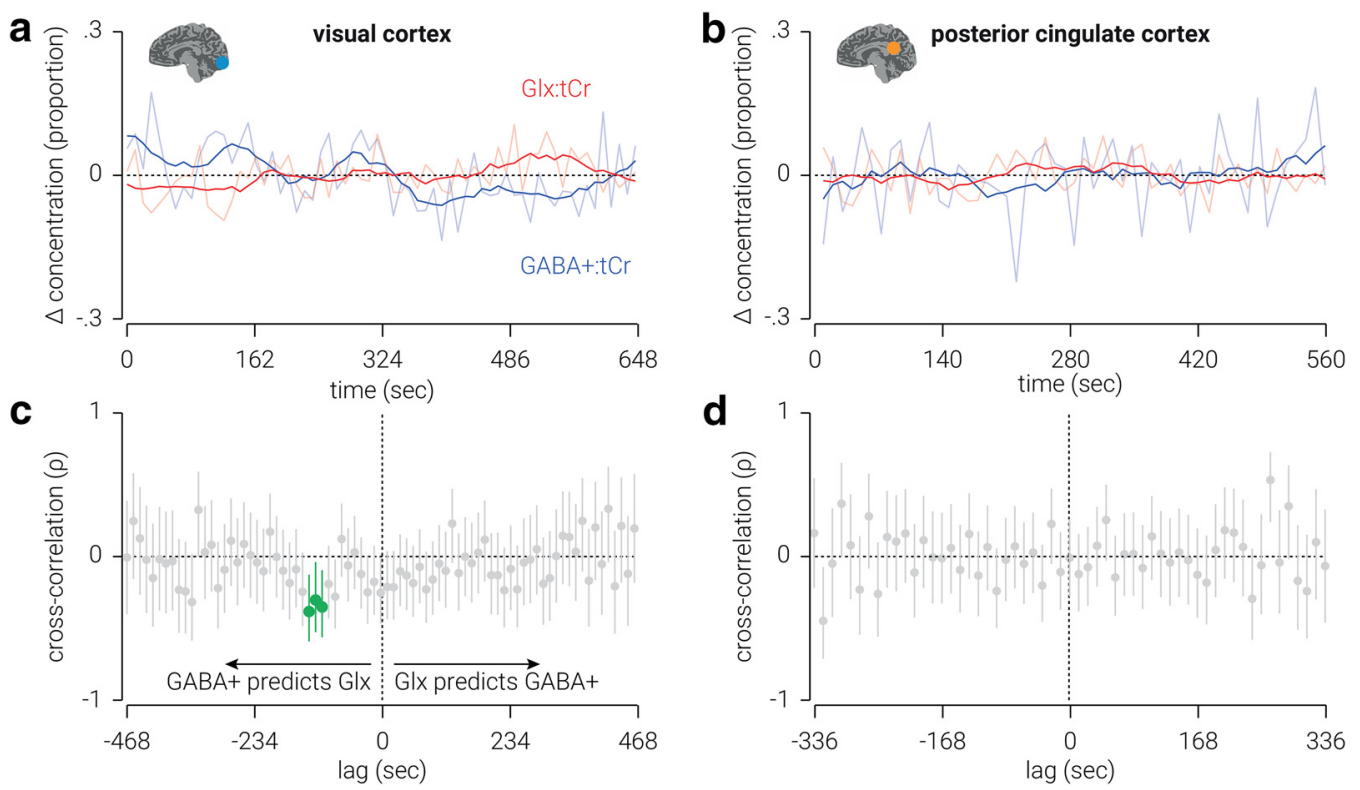

Figure 4. High-resolution temporal dynamics of neurometabolites in visual and posterior cingulate cortices. $\boldsymbol{a}$, $\boldsymbol{b}$, Changes in $\mathrm{GABA}^{+}$and Glx concentrations, proportional to the average, measured from voxels targeting visual (a) and posterior cingulate (b) cortices. Semitransparent lines indicate raw concentration estimates; for illustrational purposes, opaque lines indicate data smoothed using a window of eight time points. $\boldsymbol{c}, \boldsymbol{d}$, Cross-correlations between $\mathrm{GABA}^{+}$and Glx concentrations measured from voxels targeting visual $(\boldsymbol{c})$ and posterior cingulate $(\boldsymbol{d})$ cortices. Lag values indicate the duration between when the GABA ${ }^{+}$measurements were acquired and the Glx measurements were acquired. Correlations at negative lags indicate that GABA $^{+}$concentration predicts Glx concentration, and correlations at positive lags indicate that Glx predicts $\mathrm{GABA}^{+}$concentration. Vertical lines indicate 95\% confidence intervals; cluster-corrected correlations that are significantly different from zero are highlighted in green. All values are referenced to $\mathrm{tCr}$.

resolution analysis by applying a sliding window to the high-resolution neurometabolite trace. If the high-resolution measurements are valid, we would expect to find correspondence between the average results from the lowresolution analysis and those produced by applying a sliding window to the high-resolution measurements. For visual cortex, we found a high correspondence between the measurements produced by the two analyses for both $\mathrm{GABA}^{+}\left(n=33\right.$, Pearson $r=0.995, p=3.9 \times 10^{-33}$; Fig. $5 b$, top) and Glx $\left(n=33\right.$, Pearson $\left.r=0.995, p=5.5 \times 10^{-32}\right)$.
These results further validate the results of the high-resolution analysis in visual cortex. For posterior cingulate cortex, we found a correspondence between $\mathrm{GABA}^{+}$ measurements $\left(n=22\right.$, Pearson $r=0.655, p=9.3 \times 10^{-4}$; Fig. $5 b$, bottom), but not Glx measurements $(n=22$, Pearson $r=0.182, p=0.418$ ). The weaker correspondence for data from posterior cingulate cortex may indicate that the measurements produced by the highresolution analysis in this region are less reliable. However, it is also likely because there was less variability
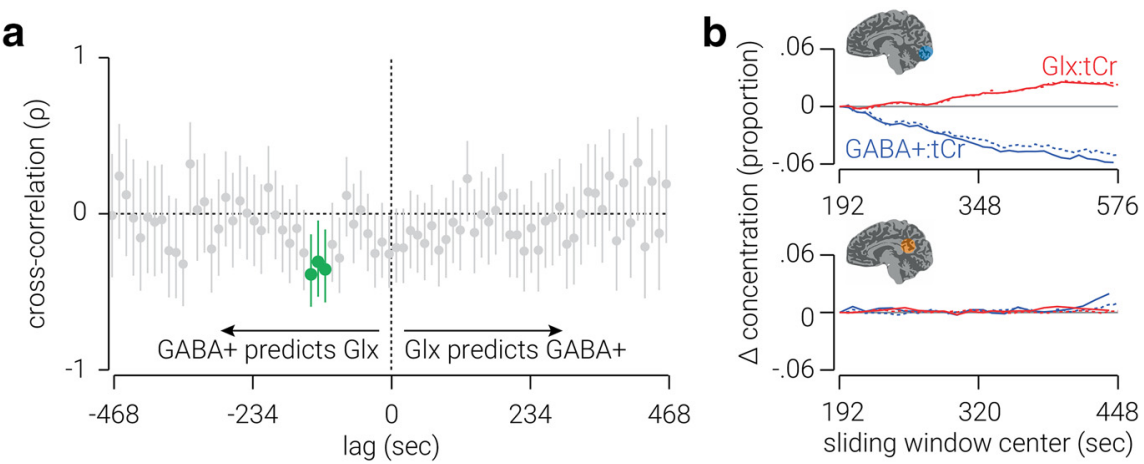

Figure 5. Controls for high-resolution analyses. a, Cross-correlations between $\mathrm{GABA}^{+}$and GIx, referenced to tNAA, measured from voxels targeting visual cortex. Vertical lines indicate 95\% confidence intervals; cluster-corrected correlations that are significantly different from zero are highlighted in green, correlations at negative lags indicate that $\mathrm{GABA}^{+}$predicts Glx, and correlations at positive lags indicate that Glx predicts $\mathrm{GABA}^{+}$. $\boldsymbol{b}$, Comparison between results from the sliding window method used in the low-temporal resolution analysis (dashed lines) and sliding window applied to results from the high-resolution analysis (solid lines) for visual (top) and posterior cingulate (bottom) cortices. 
in neurometabolite concentration in the low-resolution analysis of posterior cingulate cortex; thus, unlike in visual cortex, there may be insufficient variability to cross-validate between the two measurements.

\section{Discussion}

MRS can be used in vivo to quantify neurometabolite concentration and provide evidence for the involvement of different neurotransmitter systems (e.g., inhibitory and excitatory) in sensory and cognitive processes. In MRS studies, temporal resolution is typically sacrificed to achieve a signal-to-noise ratio sufficient to produce a reliable estimate of neurometabolite concentration. Here we use novel analyses with large datasets to reveal the dynamics of $\mathrm{GABA}^{+}$and Glx in visual and posterior cingulate cortices. We use a sliding window approach to show that when participants are at rest with their eyes closed, the concentration of $\mathrm{GABA}^{+}$and Glx in visual cortex drifts in opposite directions; that is, $\mathrm{GABA}^{+}$decreases while Glx increases over time. We then use a new method of combining MRS measurements across subjects, as opposed to time, to produce a high-temporal resolution index of neurometabolite concentration. Using this approach, we find that in visual cortex a change in the concentration of $\mathrm{GABA}^{+}$predicts the opposite change in Glx $\sim 120$ s later (e.g., an increase in $\mathrm{GABA}^{+}$predicts a later reduction in Glx).

\section{Dynamic response of GABA and GIx in visual cortex}

Several studies have investigated GABA and/or Glx/Glu concentration in visual cortex in response to different viewing conditions. Mekle et al. (2017) found an $\sim 5 \%$ reduction in GABA concentration in response to visual stimulation. By contrast, while Kurcyus et al. (2018) reported that GABA was $16 \%$ lower when participants had their eyes open with no visual stimulation compared with when they had their eyes closed, they, like others (Mangia et al., 2007; Schaller et al., 2013; Bednarõík et al., 2015, 2018), found no evidence for a difference in GABA in response to visual stimulation. Based on these somewhat inconsistent findings, one may infer that visual stimulation, or merely having the eyes open, leads to a reduction in the concentration of GABA in visual cortex. Extending this rationale, one could predict that closing the eyes should produce an increase in GABA. By contrast, we found the opposite result: during a $13 \mathrm{~min}$ period of resting in which participants' eyes were closed, the concentration of $\mathrm{GABA}^{+}$in visual cortex reduced on average by $5 \%$. These results are consistent with previous work showing that monocular deprivation leads to reduced GABA concentration $(\sim 8 \%)$ in visual cortex, but not posterior cingulate cortex, relative to a predeprivation baseline measurement (Lunghi et al., 2015). Thus, our finding that GABA is reduced when both eyes are closed may indicate that visual deprivation, either monocular or binocular, evokes a reduction in the concentration of GABA in visual cortex.

Previous observations of Glu concentration in visual cortex have been more consistent; several studies have shown Glx/Glu concentration increases (2-4\%) in response to visual stimulation (Mangia et al., 2007; Lin et al., 2012; Schaller et al., 2013; Bednarõík et al., 2015, 2018; Ip et al., 2017; Kurcyus et al., 2018). By contrast, here we found that Glx increased when participants' eyes were closed. Increased Glu in visual cortex, evoked by visual stimulation, has been linked to increased blood oxygenation level-dependent responses (Ip et al., 2017). Here we measured GIx, a complex comprising Glu and GIn, and previous $7 \mathrm{~T}$ MRS work suggests that visual stimulation evoked changes in Glu, but not Gln, in visual cortex (Schaller et al., 2013; Bednarõík et al., 2015, 2018). It is possible that the increase in Glx we found here, which occurred in the absence of visual stimulation, was driven by an alternative mechanism, one that is unrelated to BOLD activity and/or reflects changes in Gin rather than Glu. More work is needed to disambiguate changes in neurometabolite concentration that occur in visual cortex at different time scales and under different viewing conditions. For instance, future work could combine fMRI and MRS measurements to test whether the phenomenon observed here is related to BOLD activity (Ip et al., 2017). Additionally, future work could use a within-subject design to compare different voxel locations, and, separately, to assess Glu and Gln concentration drift.

Although the average concentrations of $\mathrm{GABA}^{+}$and Glx drift in opposing directions, there were some participants who showed the opposite change in neurometabolite drift. One possible explanation for this is that the concentrations of these neurometabolites oscillate at a relatively slow frequency (e.g., wavelength $=15 \mathrm{~min}$ ), and entering a state of rest (with eyes closed) introduces the linear trend of neurometabolite concentration change indicated by the average, which is summed with the larger oscillatory changes. If such oscillatory behavior was occurring, it would be unlikely to be detected in the high-resolution analysis, as troughs/peaks would be obscured by phase differences between participants. Another possible explanation for the variability between participants is that it relates to their state of wakefulness during the scan. Although subjects reported not falling asleep, it seems likely that some may have been more alert than others during the scan, and this may be related to the concentration drift observed in visual cortex. We do not have the data to examine this possibility; however, future work could concurrently collect metabolite concentration and a physiological marker of wakefulness (e.g., EEG) to assess this possibility.

Continuous unidirectional neurometabolite drift is not sustainable (i.e., neurometabolite concentration must maintain some degree of homeostasis). Thus, it seems likely that the change in concentration induced by resting only continues for a fixed period of time before stabilizing and possibly returning to "baseline" levels. Indeed, this appeared to occur for Glx, where there is no additional increase after $\sim 500 \mathrm{~s}$, and the decrease in $\mathrm{GABA}^{+}$appears to lessen after $400 \mathrm{~s}$. Future work is needed to further understand dynamic neurometabolite activity over longer periods of time.

To produce a high-temporal resolution measure of neurometabolite concentrations, we applied a novel approach in which we combined MRS transients across subjects, rather than time. This approach yields a single 
measurement of neurometabolite concentration as a function of time; thus, we cannot test the statistical significance of the changes observed. However, it is reasonable to assume that the changes estimated using sliding window or static approaches underestimate the true magnitude of change, due to averaging. The measurements produced here by combining transients across subjects provide an indication of the true magnitude of change in $\mathrm{GABA}^{+}$and Glx during the scan: up to $30 \%$ for $\mathrm{GABA}^{+}$ and $20 \%$ for Glx.

\section{Dynamic relationship between GABA and GIx}

A common finding among studies of GABA and Glu in visual cortex is that these neurometabolites change in opposite directions, and not in the same direction (Mekle et al., 2017; Kurcyus et al., 2018; Rideaux et al., 2019). This pattern would suggest an interdependence between the two neurometabolites. Given that GIn is a primary source of GABA synthesis (Paulsen et al., 1988; Patel et al., 2001; Rae et al., 2003), one may expect that a change in the concentration of GABA may result in a corresponding change in Glx, or vice versa. In line with this, our hightemporal resolution analysis of the data revealed a striking cross-correlation between these neurometabolites in visual cortex. Specifically, we found that the concentration of $\mathrm{GABA}^{+}$predicts the concentration of Glx $\sim 120$ s later. This relationship, which accounts for up to $\sim 20 \%$ of the variance of Glx, is obscured by conventional approaches of MRS analysis; indeed, we found no evidence for a relationship between the overall changes in $\mathrm{GABA}^{+}$and $\mathrm{Glx}$.

A limitation of MRS is that it measures the total concentration of neurometabolites within a localized region and cannot distinguish between intracellular and extracellular pools of GABA. It is generally thought that intracellular vesicular GABA drives neurotransmission (Belelli et al., 2009), whereas extracellular GABA maintains tonic cortical inhibition (Martin and Rimvall, 1993). Synthesis of intracellular GABA from GIn via the GABA-GIn cycle occurs on the scale of milliseconds, so it seems unlikely that this metabolic association could explain the predictive relationship between GABA and Glx concentration 120 s later. Instead, this relationship may reflect changes in the level of extracellular GABA, followed by relatively sluggish changes in the concentration of Glu to maintain the balance of inhibition and excitation.

\section{Clinical relevance}

An imbalance between excitation and inhibition, in particular between Glu and GABA, is thought to underlie a range of neurologic and psychiatric disorders including epilepsy (Bradford, 1995; Olsen and Avoli, 1997), autism (Rubenstein and Merzenich, 2003; Chao et al., 2010; Markram and Markram, 2010; Vattikuti and Chow, 2010), and schizophrenia (Kehrer et al., 2008). Here we demonstrated that it is possible to use MRS to measure changes in neurometabolite concentration at relatively high temporal resolution, and, in the process of doing so, we revealed a novel predictive relationship between $\mathrm{GABA}^{+}$and $\mathrm{Glx}$ in visual cortex of healthy human participants. More broadly, this method could be used to reveal other trends and relationships between neurometabolites that have thus far been obscured by the classic static measurement approach. Uncovering the dynamics of the excitation-inhibition balance in this way may be instrumental in understanding unhealthy brain function. However, a caveat to this approach is that it requires a relatively large amount of MRS data. Here, we used a large number of subjects to meet this requirement; however, this may not be appropriate for clinical studies in which suitable subjects are more difficult to recruit. In this case, a modified event-related version of the method used here may be effective (Branzoli et al., 2013). That is, events (e.g., a 2 min stimulus presentation) could be repeated multiple times over a long period, separated by baseline resting intervals. Transients could then be aligned according to the time course of the event and averaged together. This would provide the same signal-to-noise improvement reached in the current study, while requiring fewer participants.

\section{Conclusion}

The relatively low signal-to-noise ratio of MRS measurements has shaped the types of questions that the technique has been used to address. Here we overcome this limitation by combining data from large cohorts to examine the dynamics of GABA and Glx concentrations in visual cortex. Through use of existing and novel analyses, we reveal opposing dynamic shifts in GABA and Glx in visual cortex while participants are at rest. Further, we demonstrate a predictive relationship between GABA and Glx in visual cortex. This study exposes temporal trends of primary neurotransmitters in visual cortex, and more generally, these findings demonstrate the feasibility of using MRS to investigate in vivo dynamic changes of neurometabolites.

\section{References}

Bednarõík P, Tkáč I, Giove F, Dinuzzo M, Deelchand DK, Emir UE, Eberly LE, Mangia S (2015) Neurochemical and BOLD responses during neuronal activation measured in the human visual cortex at 7 Tesla. J Cereb Blood Flow Metab 35:601-610.

Bednarõík P, Tkáč I, Giove F, Eberly LE, Deelchand DK, Barreto FR, Mangia $S$ (2018) Neurochemical responses to chromatic and achromatic stimuli in the human visual cortex. J Cereb Blood Flow Metab 38:347-359.

Belelli D, Harrison NL, Maguire J, Macdonald RL, Walker MC, Cope

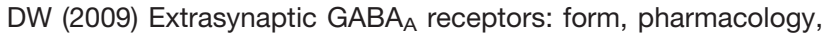
and function. J Neurosci 29:12757-12763.

Bhattacharyya PK, Lowe MJ, Phillips MD (2007) Spectral quality control in motion-corrupted single-voxel J-difference editing scans: an interleaved navigator approach. Magn Reson Med 58:808-812.

Bonett DG, Wright TA (2000) Sample size requirements for estimating Pearson, Kendall and Spearman correlations. Psychometrika 65:23-28.

Boroojerdi B, Bushara KO, Corwell B, Immisch I, Battaglia F, Muellbacher W, Cohen LG (2000) Enhanced excitability of the human visual cortex induced by short-term light deprivation. Cereb Cortex 10:529-534.

Bradford HF (1995) Glutamate, GABA and epilepsy. Prog Neurobiol 47:477-511. 
Branzoli F, Techawiboonwong A, Kan H, Webb A, Ronen I (2013) Functional diffusion-weighted magnetic resonance spectroscopy of the human primary visual cortex at $7 \mathrm{~T}$. Magn Reson Med 69:303-309.

Chao HT, Chen H, Samaco RC, Xue M, Chahrour M, Yoo J, Neul JL, Gong S, Lu HC, Heintz N, Ekker M, Rubenstein JLR, Noebels JL, Rosenmund C, Zoghbi HY (2010) Dysfunction in GABA signalling mediates autism-like stereotypies and Rett syndrome phenotypes. Nature 468:263-269.

Chen C, Sigurdsson HP, Pépés SE, Auer DP, Morris PG, Morgan PS, Gowland PA, Jackson SR (2017) Activation induced changes in GABA: functional MRS at $7 \mathrm{~T}$ with MEGA-sLASER. Neuroimage 156:207-213.

Dong Z (2015) Proton MRS and MRSI of the brain without water suppression. Prog Nucl Magn Reson Spectrosc 86-87:65-79.

Edden RAE, Puts NAJ, Harris AD, Barker PB, Evans CJ (2014) Gannet: a batch-processing tool for the quantitative analysis of gamma-aminobutyric acid-edited MR spectroscopy spectra. J Magn Reson Imaging 40:1445-1452.

Harris AD, Puts NAJ, Edden RAE (2015) Tissue correction for GABAedited MRS: considerations of voxel composition, tissue segmentation, and tissue relaxations. J Magn Reson Imaging 42:14311440.

Ip IB, Berrington A, Hess AT, Parker AJ, Emir UE, Bridge H (2017) Combined fMRI-MRS acquires simultaneous glutamate and BOLD-fMRI signals in the human brain. Neuroimage 155:113-119.

Jansen JFA, Backes WH, Nicolay K, Kooi ME (2006) 1H MR spectroscopy of the brain: absolute quantification of metabolites. Radiology 240:318-332.

Kehrer C, Maziashvili N, Dugladze T, Gloveli T (2008) Altered excitatory-inhibitory balance in the NMDA-hypofunction model of schizophrenia. Front Mol Neurosci 1:6.

Kurcyus K, Annac E, Hanning NM, Harris AD, Oeltzschner G, Edden RAE, Riedl V (2018) Opposite dynamics of GABA and glutamate levels in the occipital cortex during visual processing. J Neurosci 38:9967-9976.

Lange T, Zaitsev M, Buechert M (2011) Correction of frequency drifts induced by gradient heating in $1 \mathrm{H}$ spectra using interleaved reference spectroscopy. J Magn Reson Imaging 33:748-754.

Lin Y, Stephenson MC, Xin L, Napolitano A, Morris PG (2012) Investigating the metabolic changes due to visual stimulation using functional proton magnetic resonance spectroscopy at $7 \mathrm{~T}$. J Cereb Blood Flow Metab 32:1484-1495.

Lunghi C, Emir UE, Morrone MC, Bridge H (2015) Short-term monocular deprivation alters GABA in the adult human visual cortex. Curr Biol 25:1496-1501.

Mangia S, Tkáč I, Gruetter R, Van de Moortele P-F, Maraviglia B, Uğurbil K (2007) Sustained neuronal activation raises oxidative metabolism to a new steady-state level: evidence from ${ }^{1} \mathrm{H}$ NMR spectroscopy in the human visual cortex. J Cereb Blood Flow Metab 27:1055-1063.

Markram K, Markram H (2010) The intense world theory-a unifying theory of the neurobiology of autism. Front Hum Neurosci 4:224.

Martin DL, Rimvall K (1993) Regulation of $\gamma$-aminobutyric acid synthesis in the brain. J Neurochem 60:395-407.

Mekle R, Kühn S, Pfeiffer H, Aydin S, Schubert F, Ittermann B (2017) Detection of metabolite changes in response to a varying visual stimulation paradigm using short-TE ${ }^{1} \mathrm{H}$ MRS at $7 \mathrm{~T}$. NMR Biomed 30:e3672.
Mescher M, Tannus A, Johnson MO, Garwood M (1996) Solvent suppression using selective echo dephasing. J Magn Reson A 123:226-229.

Mescher M, Merkle H, Kirsch J, Garwood M, Gruetter R (1998) Simultaneous in vivo spectral editing and water suppression. NMR Biomed 11:266-272.

Mikkelsen M, Barker PB, Bhattacharyya PK, Brix MK, Buur PF, Cecil KM, Chan KL, Chen DY-T, Craven AR, Cuypers K, Dacko M, Duncan NW, Dydak U, Edmondson DA, Ende G, Ersland L, Gao F, Greenhouse I, Harris AD, He N, et al. (2017) Big GABA: edited MR spectroscopy at 24 research sites. Neuroimage 159:32-45.

Mikkelsen M, Loo RS, Puts NAJ, Edden RAE, Harris AD (2018) Designing GABA-edited magnetic resonance spectroscopy studies: considerations of scan duration, signal-to-noise ratio and sample size. J Neurosci Methods 303:86-94.

Mikkelsen M, Rimbault DL, Barker PB, Bhattacharyya PK, Brix MK, Buur PF, Cecil KM, Chan KL, Chen DY-T, Craven AR, Cuypers K, Dacko M, Duncan NW, Dydak U, Edmondson DA, Ende G, Ersland L, Forbes MA, Gao F, Greenhouse I, et al. (2019) Big GABA II: water-referenced edited MR spectroscopy at 25 research sites. Neuroimage 191:537-548.

Mullins PG, McGonigle DJ, O'Gorman RL, Puts NAJ, Vidyasagar R, Evans CJ, Edden RAE (2014) Current practice in the use of MEGAPRESS spectroscopy for the detection of GABA. Neuroimage 86:43-52.

Olsen RW, Avoli M (1997) GABA and Epileptogenesis. Epilepsia 38:399-407.

Patel AB, Rothman DL, Cline GW, Behar KL (2001) Glutamine is the major precursor for GABA synthesis in rat neocortex in vivo following acute GABA-transaminase inhibition. Brain Res 919:207-220.

Paulsen RE, Odden E, Fonnum F (1988) Importance of glutamine for gamma-aminobutyric acid synthesis in rat neostriatum in vivo. $\mathrm{J}$ Neurochem 51:1294-1299.

Rae C, Hare N, Bubb WA, McEwan SR, Bröer A, McQuillan JA, Balcar VJ, Conigrave AD, Bröer S (2003) Inhibition of glutamine transport depletes glutamate and GABA neurotransmitter pools: further evidence for metabolic compartmentation. J Neurochem 85:503-514.

Rideaux R, Welchman AE (2018) Proscription supports robust perceptual integration by suppression in human visual cortex. Nat Commun 9:1502.

Rideaux R, Goncalves NR, Welchman AE (2019) Mixed-polarity random-dot stereograms alter GABA and Glx concentration in the early visual cortex. J Neurophysiol 122:888-896.

Rubenstein JLR, Merzenich MM (2003) Model of autism: increased ratio of excitation/inhibition in key neural systems. Genes Brain Behav 2:255-267.

Schaller B, Mekle R, Xin L, Kunz N, Gruetter R (2013) Net increase of lactate and glutamate concentration in activated human visual cortex detected with magnetic resonance spectroscopy at 7 tesla. $J$ Neurosci Res 91:1076-1083.

Tkáč I, Gruetter R (2005) Methodology of 1H NMR spectroscopy of the human brain at very high magnetic fields. Appl Magn Reson 29:139-157.

Vattikuti S, Chow CC (2010) A computational model for cerebral cortical dysfunction in autism spectrum disorders. Biol Psychiatry 67:672-678. 